\title{
LYAPUNOV STABILITY SOLUTIONS OF FRACTIONAL INTEGRODIFFERENTIAL EQUATIONS
}

\author{
SHAHER MOMANI and SAMIR HADID
}

Received 28 December 2003

\begin{abstract}
Lyapunov stability and asymptotic stability conditions for the solutions of the fractional integrodiffrential equations $x^{(\alpha)}(t)=f(t, x(t))+\int_{t_{0}}^{t} K(t, s, x(s)) d s, 0<\alpha \leq 1$, with the initial condition $x^{(\alpha-1)}\left(t_{0}\right)=x_{0}$, have been investigated. Our methods are applications of Gronwall's lemma and Schwartz inequality.
\end{abstract}

2000 Mathematics Subject Classification: 26A33, 34D20.

1. Introduction. Consider the fractional integrodiffrential equations of the type

$$
x^{(\alpha)}(t)=f(t, x(t))+\int_{t_{0}}^{t} K(t, s, x(s)) d s, \quad 0<\alpha \leq 1,
$$

with the initial condition

$$
x^{(\alpha-1)}\left(t_{0}\right)=x_{0}
$$

where $\mathbb{R}$ is the set of real numbers, $J=\left[t_{0}, t_{0}+a\right], f \in C\left[J \times \mathbb{R}^{n}, \mathbb{R}^{n}\right]$, and $K \in C[J \times$ $\left.J \times \mathbb{R}^{n}, \mathbb{R}^{n}\right]$, where $\mathbb{R}^{n}$ denotes the real $n$-dimensional Euclidean space, and $x_{0}$ is a real constant.

The existence and uniqueness of solution of fractional differential equations, when the integral part in (1.1) is identically zero, has been investigated by some authors, see $[1,3,5,6]$.

In recent papers $[7,8]$, we used Schauder's fixed-point theorem to obtain local existence, and Tychonov's fixed-point theorem to obtain global existence of solution of the fractional integrodifferential equations (1.1) and (1.2). The existence of extremal (maximal and minimal) solutions of the fractional integrodiffrential equations (1.1) and (1.2) using comparison principle and Ascoli lemma has been investigated in [9].

In this paper, we are concerned with the stability and asymptotic stability, in the sense of Lyapunov, for the solution of the fractional integrodiffrential equations (1.1) and (1.2). We will assume that $f(t, 0) \equiv 0$ and $K(t, s(t), 0) \equiv 0$ for all $t \in J$, so that $x=0$ is a solution of (1.1).

The zero solution is said to be stable (in the sense of Lyapunov) if, given $\varepsilon>0$, there exists $\delta>0$ such that any solution $x(t)$ of (1.1) satisfying $\left|x\left(t_{0}\right)\right|<\delta$ for $t=t_{0}$ also satisfies $|x(t)|<\varepsilon$ for all $t \geq t_{0}$. The zero solution is said to be asymptotically stable if, in addition to being stable, $|x(t)| \rightarrow 0$ as $t \rightarrow \infty$. 
Our result is a generalization of Hadid and Alshamani [4], in which it was shown that under certain conditions on $f$ the zero solution of the initial value problem (IVP):

$$
x^{(\alpha)}(t)=f(t, x(t)), \quad \alpha \in \mathbb{R}, 0<\alpha \leq 1,
$$

with the initial condition $x^{(\alpha-1)}\left(t_{0}\right)=x_{0}$ is stable and hence it is asymptotically stable.

Next we set forth definitions and lemmas to be used in this paper. For proofs and details see $[1,2,3]$.

DEFINITION 1.1. Let $f$ be a function which is defined a.e. on $[a, b]$. For $\alpha>0$, define

$$
\stackrel{b}{I}^{\alpha} f=\frac{1}{\Gamma(\alpha)} \int_{a}^{b}(b-s)^{\alpha-1} f(s) d s,
$$

provided that this integral (Lebesgue) exists, where $\Gamma$ is the Gamma function.

LEMMA 1.2. The IVP (1.1) and (1.2) is equivalent to the nonlinear integral equation

$$
\begin{aligned}
x(t)= & \frac{x_{0}}{\Gamma(\alpha)}\left(t-t_{0}\right)^{\alpha-1}+\frac{1}{\Gamma(\alpha)} \int_{t_{0}}^{t}(t-s)^{\alpha-1} f(s, x(s)) d s \\
& +\frac{1}{\Gamma(\alpha)} \int_{t_{0}}^{t}(t-s)^{\alpha-1} \int_{s}^{t} K(\sigma, s, x(s)) d \sigma d s,
\end{aligned}
$$

where $0<t_{0}<t \leq t_{0}+a$. In other words, every solution of the integral (1.5) is also a solution of the original IVP (1.1) and (1.2), and vice versa.

Proof. It can be proved easily by applying the integral operator (1.4) with $a=t_{0}$ and $b=t$ to both sides of (1.1), as we did in [4], and using some classical results from fractional calculus in [2] to get (1.5).

LEMMA 1.3 (Gronwall's lemma). Let $u(t)$ and $v(t)$ be nonnegative continuous functions on some interval $t_{0} \leq t \leq t_{0}+a$. Also, let the function $f(t)$ be positive, continuous, and monotonically nondecreasing on $t_{0} \leq t \leq t_{0}+a$ and satisfy the inequality

$$
u(t) \leq f(t)+\int_{t_{0}}^{t} u(s) v(s) d s
$$

then, there exists

$$
u(t) \leq f(t) \exp \left(\int_{t_{0}}^{t} v(s) d s\right) \quad \text { for } t_{0} \leq t \leq t_{0}+a .
$$

Proof. For the proof of Lemma 1.3, see [10].

2. Stability conditions. In this section, we will prove our main results, and discuss the stability and asymptotic stability of the solution of (1.1) satisfying (1.2).

THEOREM 2.1. Let the function $f$ satisfy the inequality

$$
|f(t, x(t))| \leq \gamma(t)|x|,
$$


and let $K$ satisfy

$$
\left|\int_{s}^{t} K(\sigma, s, x(s)) d \sigma\right| \leq \delta(t)|x|, \quad s \in\left[t_{0}, t\right],
$$

where $\gamma(t)$ and $\delta(t)$ are continuous nonnegative functions such that

$$
\sup \int_{t_{0}}^{t}(t-s)^{\alpha-1}[\gamma(s)+\delta(s)] d s<\infty .
$$

Then every solution $x(t)$ of (1.1) satisfies

$$
|x(t)| \leq \frac{\left|x_{0}\right|}{\Gamma(\alpha)}\left(t-t_{0}\right)^{\alpha-1} \exp \left\{\frac{1}{\Gamma(\alpha)} \int_{t_{0}}^{t}(t-s)^{\alpha-1}[\gamma(s)+\delta(s)] d s\right\}<\infty .
$$

Proof. For $0 \leq t_{0}<s<t \leq t_{0}+a$, it follows from (1.5) that

$$
\begin{aligned}
\Gamma(\alpha)|x(t)| \leq & \left|x_{0}\right|\left(t-t_{0}\right)^{\alpha-1} \\
& +\int_{t_{0}}^{t}(t-s)^{\alpha-1} \gamma(s)|x(s)| d s \\
& +\int_{t_{0}}^{t}(t-s)^{\alpha-1} \delta(s)|x(s)| d s .
\end{aligned}
$$

By combining the integrals on the right-hand side, we get

$$
\begin{aligned}
\Gamma(\alpha)|x(t)| \leq & \left|x_{0}\right|\left(t-t_{0}\right)^{\alpha-1} \\
& +\frac{1}{\Gamma(\alpha)} \int_{t_{0}}^{t}(t-s)^{\alpha-1}[\gamma(s)+\delta(s)] \Gamma(\alpha)|x(s)| d s .
\end{aligned}
$$

By Gronwell's lemma, we obtain

$$
\Gamma(\alpha)|x(t)| \leq\left|x_{0}\right|\left(t-t_{0}\right)^{\alpha-1} \exp \left\{\frac{1}{\Gamma(\alpha)} \int_{t_{0}}^{t}(t-s)^{\alpha-1}[\gamma(s)+\delta(s)] d s\right\} .
$$

Therefore

$$
|x(t)| \leq \frac{\left|x_{0}\right|\left(t-t_{0}\right)^{\alpha-1}}{\Gamma(\alpha)} \exp \left\{\frac{1}{\Gamma(\alpha)} \int_{t_{0}}^{t}(t-s)^{\alpha-1}[\gamma(s)+\delta(s)] d s\right\} .
$$

Hence the theorem is proved.

COROLLARY 2.2. If

$$
\int_{t_{0}}^{t}(t-s)^{\alpha-1}[\gamma(s)+\delta(s)] d s=O\left(\left(t-t_{0}\right)^{\alpha-1}\right),
$$

then

$$
|x(t)| \leq C_{0}\left(\left(t-t_{0}\right)^{\alpha-1}\right),
$$

where $C_{0}$ is a positive constant, and hence the solution of (1.1) and (1.2) is asymptotically stable. 
COROLLARY 2.3. It can easily be shown from (2.10) that

$$
x(t) \in L^{2}\left(t_{0}, \infty\right) \quad \forall 0<\alpha<\frac{1}{2} .
$$

Next, we will prove another important stability result. The result is in connection with $\alpha$; the method we will use is an application of Schwartz inequality.

\section{THEOREM 2.4. Assume that}

(i) the function $f$ is in $L^{2}\left(t_{0}, \infty\right)$ as a function of $t$,

(ii) $K(t, s, x)=O\left((t-s)^{\alpha-3 / 2}\right)$.

Then, for $0<\alpha<1 / 2$, the zero solution of (1.1) and (1.2) is asymptotically stable.

Proof. For $0 \leq t_{0}<s<t \leq t_{0}+a$, it follows from (1.5) that

$$
\begin{aligned}
\Gamma(\alpha) x(t)= & x_{0}\left(t-t_{0}\right)^{\alpha-1}+\int_{t_{0}}^{t}(t-s)^{\alpha-1} f(s, x(s)) d s \\
& +\int_{t_{0}}^{t}(t-s)^{\alpha-1} \int_{s}^{t} K(\sigma, s, x(s)) d \sigma d s .
\end{aligned}
$$

By applying the absolute value, we get

$$
\begin{aligned}
\Gamma(\alpha)|x(t)| \leq & \left|x_{0}\right|\left(t-t_{0}\right)^{\alpha-1}+\int_{t_{0}}^{t}(t-s)^{\alpha-1}|f(s, x(s))| d s \\
& +\int_{t_{0}}^{t}(t-s)^{\alpha-1} \int_{s}^{t}|K(\sigma, s, x(s))| d \sigma d s .
\end{aligned}
$$

By Schwartz inequality, we obtain

$$
\begin{aligned}
\Gamma(\alpha)|x(t)| \leq & \left|x_{0}\right|\left(t-t_{0}\right)^{\alpha-1} \\
& +\left(\int_{t_{0}}^{t}(t-s)^{2 \alpha-2} d s\right)^{1 / 2}\left(\int_{t_{0}}^{t}|f(s, x(s))|^{2} d s\right)^{1 / 2} \\
& +\left(\int_{t_{0}}^{t}(t-s)^{2 \alpha-2} d s\right)^{1 / 2}\left(\int_{t_{0}}^{t}\left(\int_{s}^{t}|K(\sigma, s, x(s))| d \sigma\right)^{2} d s\right)^{1 / 2} .
\end{aligned}
$$

Now, using (i) and (ii) in the statement of the theorem and integrating, we obtain

$$
\Gamma(\alpha)|x(t)| \leq\left|x_{0}\right|\left(t-t_{0}\right)^{\alpha-1}+C_{1}\left(t-t_{0}\right)^{\alpha-1 / 2}+C_{2}\left(t-t_{0}\right)^{\alpha-1 / 2},
$$

where $C_{1}$ and $C_{2}$ are positive constants (we can calculate them easily).

By (2.15), and for $0 \leq t_{0}<s<t \leq t_{0}+a$, we have

$$
|x(t)| \leq\left(t-t_{0}\right)^{\alpha-1 / 2}\left[\frac{\left|x_{0}\right|}{\Gamma(\alpha)}\left(t-t_{0}\right)^{-1 / 2}+C_{1}+C_{2}\right]
$$

This implies that the zero solution of (1.1) and (1.2) is asymptotically stable. Hence the theorem is proved. 


\section{REFERENCES}

[1] M. A. Al-Bassam, Some existence theorems on differential equations of generalized order, J. reine angew. Math. 218 (1965), 70-78.

[2] J. H. Barrett, Differential equations of non-integer order, Canadian J. Math. 6 (1954), 529541.

[3] S. B. Hadid, Local and global existence theorems on differential equations of non-integer order, J. Fract. Calc. 7 (1995), 101-105.

[4] S. B. Hadid and J. G. Alshamani, Liapunov stability of differential equations of noninteger order, Arab J. Math. 7 (1986), no. 1-2, 5-17.

[5] S. B. Hadid, B. Masaedeh, and S. Momani, On the existence of maximal and minimal solutions of differential equations of non-integer order, J. Fract. Calc. 9 (1996), 41-44.

[6] S. B. Hadid, A. A. Ta'ani, and S. M. Momani, Some existence theorems on differential equations of generalized order through a fixed-point theorem, J. Fract. Calc. 9 (1996), 45-49.

[7] S. M. Momani, Local and global existence theorems on fractional integro-differential equations, J. Fract. Calc. 18 (2000), 81-86.

[8] _ Some existence theorems on fractional integro-differential equations, Abhath AlYarmouk Journal 10 (2001), 435-444.

[9] S. M. Momani and R. El-Khazali, On the existence of extremal solutions of fractional integrodifferential equations, J. Fract. Calc. 18 (2000), 87-92.

[10] O. Plaat, Ordinary Differential Equations, Holden-Day Series in Mathematics, Holden-Day, San Francisco, 1971.

Shaher Momani: Department of Mathematics, Faculty of Science, Mutah University, P.O. Box 7, Al-Karak, Jordan

E-mail address: shahermm@yahoo.com

Samir Hadid: Department of Mathematics and Science, Faculty of Education and Basic Science, Ajman University of Science and Technology Network, P.O. Box 346, Ajman, UAE

E-mail address: sbhadid@yahoo.com 


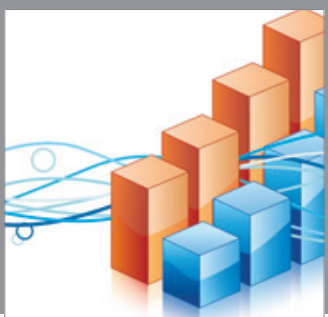

Advances in

Operations Research

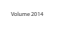

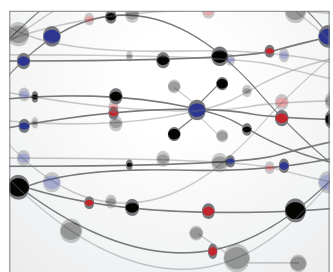

\section{The Scientific} World Journal
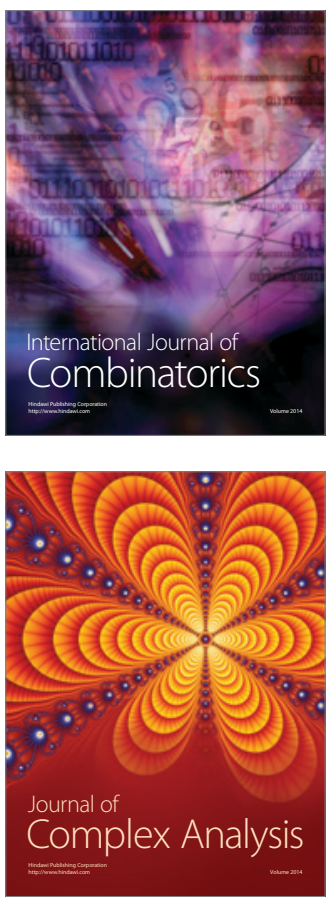

International Journal of

Mathematics and

Mathematical

Sciences
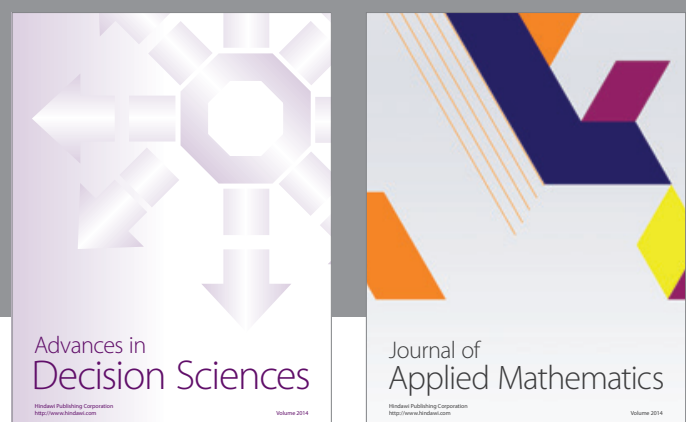

Journal of

Applied Mathematics
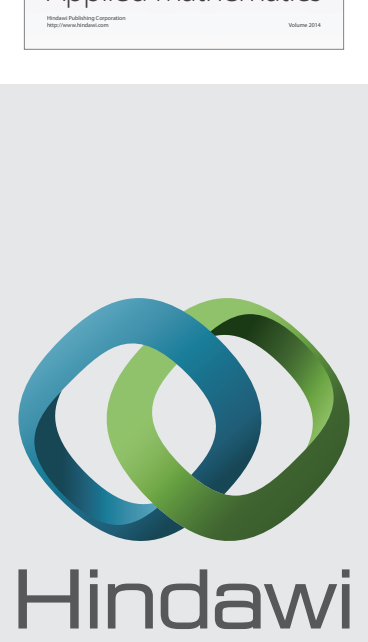

Submit your manuscripts at http://www.hindawi.com
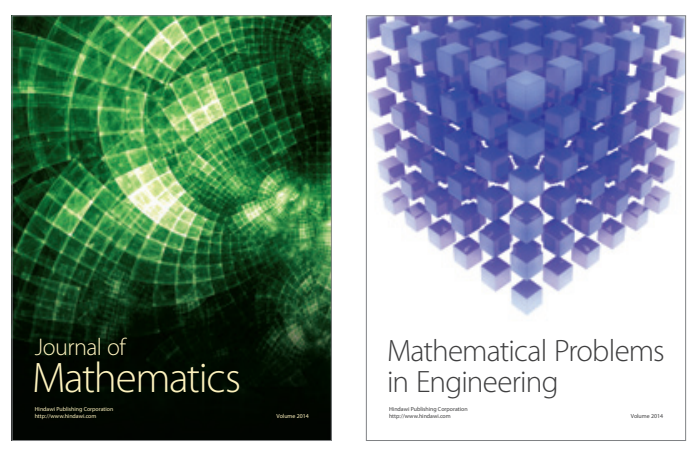

Mathematical Problems in Engineering
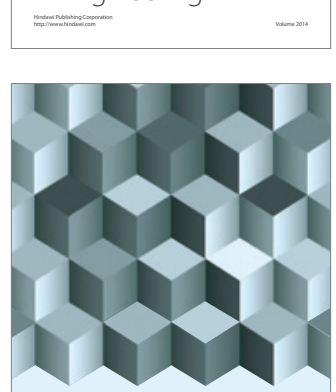

Journal of

Function Spaces
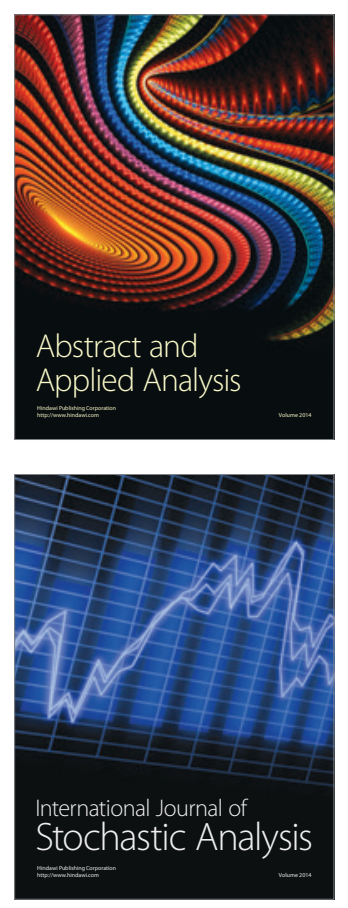

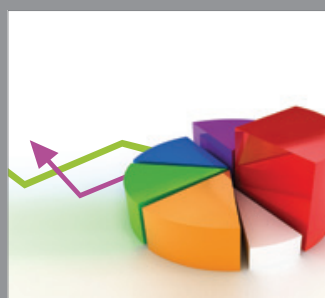

ournal of

Probability and Statistics

Promensencen
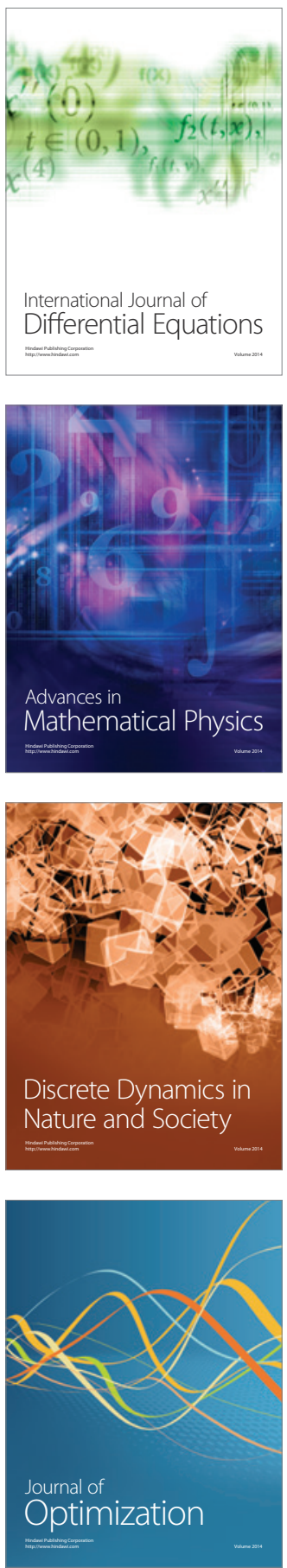